\section{Raquel da Rocha Paiva Maia ' \\ Victor Wünsch Filho"}

\title{
Infection and childhood leukemia: review of evidence
}

\section{Infecção e leucemia infantil: revisão das evidências}

\begin{abstract}
OBJECTIVE: To analyze studies that evaluated the role of infections as well as indirect measures of exposure to infection in the risk of childhood leukemia, particularly acute lymphoblastic leukemia.
\end{abstract}

\begin{abstract}
METHODS: A search in Medline, Lilacs, and SciELO scientific publication databases initially using the descriptors "childhood leukemia" and "infection" and later searching for the words "childhood leukemia" and "maternal infection or disease" or "breastfeeding" or "daycare attendance" or "vaccination" resulted in 62 publications that met the following inclusion criteria: subject aged $\leq 15$ years; specific analysis of cases diagnosed with acute lymphoblastic leukemia or total leukemia; exposure assessment of mothers' or infants' to infections (or proxy of infection), and risk of leukemia.
\end{abstract}

RESULTS: Overall, 23 studies that assessed infections in children support the hypothesis that occurrence of infection during early childhood reduces the risk of leukemia, but there are disagreements within and between studies. The evaluation of exposure to infection by indirect measures showed evidence of reduced risk of leukemia associated mainly with daycare attendance. More than $50.0 \%$ of the 16 studies that assessed maternal exposure to infection observed increased risk of leukemia associated with episodes of influenza, pneumonia, chickenpox, herpes zoster, lower genital tract infection, skin disease, sexually transmitted diseases, Epstein-Barr virus, and Helicobacter pylori.

CONCLUSIONS: Although no specific infectious agent has been identified, scientific evidence suggests that exposure to infections has some effect on

Programa de Pós-Graduação em Saúde Pública. Faculdade de Saúde Pública. Universidade de São Paulo. São Paulo, SP, Brasil

childhood leukemia etiology.

DESCRIPTORS: Children. Leukemia, etiology. Causality. Infection.

Departamento de Epidemiologia. Faculdade de Saúde Pública. Universidade de São Paulo. São Paulo, SP, Brasil

\section{Review.}

Correspondence:

Raquel da Rocha Paiva Maia

Rua Roque Garcia, 363 Loteamento

Santo Afonso

69908-836 Rio Branco, AC, Brasil

E-mail: raquelmaia@usp.br 


\section{RESUMO}

OBJETIVO: Analisar estudos que avaliaram o papel de infecções e de medidas indiretas de exposição às infecções no risco de leucemia infantil, principalmente da leucemia linfocítica aguda.

MÉTODOS: A busca nas bases de dados Medline, Lilacs e SciELO utilizando-se inicialmente os descritores "leucemia infantil" e "infecção" e, posteriormente, pesquisando-se as palavras "leucemia infantil" e "infecção ou doença materna" ou "aleitamento materno" ou "frequência à creche" ou "vacinação" recuperou 62 publicações que atenderam aos seguintes critérios de inclusão: amostra composta por sujeitos com idade inferior ou igual a 15 anos; análise específica de casos diagnosticados com leucemia linfocítica aguda ou todas as leucemias; avaliação de exposição materna ou infantil a infecções (ou medidas indiretas de exposição à infecção) e risco de leucemia.

RESULTADOS: Globalmente, os 23 estudos que avaliaram infecções nas crianças suportam a hipótese de que a ocorrência de infecções no início da infância reduz o risco de leucemia, mas existem discordâncias intra e entre estudos. A avaliação por meio das medidas indiretas de exposição à infecção mostrou evidências de redução do risco de leucemia associado principalmente com frequência à creche. Mais de 50,0\% dos 16 estudos que avaliaram exposição materna à infecção observaram aumento do risco de leucemia associado com episódios de gripe, pneumonia, varicela, herpes zoster, infecção do trato genital inferior, doença de pele, doenças sexualmente transmissíveis, vírus Epstein-Barr (EBV) e Helicobacter pylori.

CONCLUSÕES: Embora nenhum agente infeccioso específico tenha sido identificado, as evidências científicas são sugestivas de que a exposição a infecções interfere na etiologia da leucemia infantil.

DESCRITORES: Crianças. Leucemia, etiologia. Causalidade. Infecção. Revisão.

\section{INTRODUCTION}

Leukemia makes up about one third of all malignancies in the 0-14 year old age group. The most common subtype, acute lymphoblastic leukemia (ALL), represents about $80.0 \%$ of these cases. ${ }^{57}$ The incidence rate of ALL was estimated as 35.2 per million children aged under 15 in Brazil, and children under five are the most affected. ${ }^{60}$

Potential risk factors for childhood leukemia (CL) are conflicting. Exposure to ionizing radiation, commonly accepted as a cause of leukemia, does not explain all cases of leukemia in children. ${ }^{5}$ The etiology of CL has a multifactorial character. Leukemic cells that carry genetic alterations arise mainly before birth. Translocation between chromosomes 12 and 21 causes fusion of the TEL and AML1 gene; producing aberrant proteins that inhibit gene activity and change the capacity for self-renewal and differentiation of hematopoietic stem cells; this change represents the most common structural genetic abnormality in children with leukemia. ${ }^{45,74}$ About $1.0 \%$ of healthy newborns have this translocation and one leukemic clone generated in the uterus, however, they do not develop disease. ${ }^{45}$ This fact and the low correlation between identical twin ALL (about 5,0\% in infants 2-6 years) suggests that the disease probably begins in uterus, but a postnatal event is required for it to develop; this is known as a "two hit model". ${ }^{16,17}$ Studies have tested whether infections may be involved in the etiology of CL. Three main hypothesis can explain the impact infections have on the disease development.

Smith ${ }^{76}$ suggested that infection during pregnancy allows the infectious agent to be transmitted to the fetus and cause genetic instability, which leads to increased risk of developing c-ALL (B-cell precursor of common ALL) up to five years of age. This proposal would explain the peak occurrence of ALL in the 2-5 year old age group.

The hypothesis of "delayed infection", proposed by Greaves ${ }^{17,19}$ suggests that the occurrence of common infections early in life may play a protective role against 
Table 1. Review of studies on maternal infection and risk of childhood leukemia, 1973-2011.

\begin{tabular}{|c|c|c|c|c|c|}
\hline Reference & Place & $\begin{array}{l}\text { Study } \\
\text { design }\end{array}$ & Infectious agent or disease & Disease & Risk $(95 \% \mathrm{Cl})$ \\
\hline \multicolumn{6}{|l|}{ Increased risk } \\
\hline Hakulinen et $\mathrm{al}^{21}(1973)$ & Finland & $\mathrm{BC}$ & Asian influenza & $\mathrm{CL}$ & $\begin{array}{c}\text { Exposed incidence: } 68.1 / \text { million } \\
\text { Unexposed incidence: } \\
4.2 / \text { million; } P=0.048\end{array}$ \\
\hline Austin et $\mathrm{al}^{2}(1975)$ & Los Angeles & $\mathrm{BC}$ & Influenza & $\mathrm{CL}$ & RR 3.4 \\
\hline Vianna \& Polan ${ }^{83}$ (1976) & USA & $\mathrm{ECO}$ & Chicken pox & $\mathrm{CL}$ & $\begin{array}{l}\text { Expected incidence }<1 \\
\text { Observed incidence }=3\end{array}$ \\
\hline Till et $\mathrm{al}^{78}(1979)$ & England & $\mathrm{CC}$ & Varicella and herpes zoster & ALL & $\begin{array}{l}\text { Number cases higher than } \\
\text { expected ( } 2 \text { of } 54)\end{array}$ \\
\hline Mckinney et $\mathrm{al}^{43}$ (1987) & IREESC & $\mathrm{CC}$ & Skin disease & $\mathrm{CL}$ & RR $2.3(1.1 ; 4.7)$ \\
\hline Buckley et al ${ }^{8}$ (1994) & USA Canada & $\mathrm{CC}$ & Any maternal infection & ALL & OR $1.5(p<0.05)$ \\
\hline Naumburg et $\mathrm{al}^{47}$ (2002) & Sweden & $\mathrm{CC}$ & $\begin{array}{l}\text { Lower genital tract } \\
\text { infection }\end{array}$ & $\begin{array}{c}\mathrm{ALL} \\
\mathrm{CL}\end{array}$ & $\begin{array}{l}\text { OR } 1.63(1.04 ; 2.53) \\
\text { OR } 1.78(1.17 ; 2.72)\end{array}$ \\
\hline Lehtinen et $\mathrm{al}^{35}$ (2003) & Finland Iceland & $\mathrm{CC}$ & Epstein-Barr virus & ALL & OR $2.9(1.5 ; 5,8)$ \\
\hline Lehtinen et $\mathrm{al}^{36}(2005)$ & Finland Iceland & $\mathrm{CC}$ & Helicobacter pylori & $\mathrm{CL}$ & OR $2.8(1.1 ; 6.9)$ \\
\hline Kwan et $\mathrm{al}^{33}$ (2007) & California & $\mathrm{CC}$ & $\begin{array}{l}\text { Influenza/pneumonia } \\
\text { Sexually transmitted } \\
\text { diseases }\end{array}$ & $\begin{array}{l}\text { ALL } \\
C L \\
\mathrm{ALL} \\
\mathrm{CL}\end{array}$ & $\begin{array}{l}\text { OR } 1.89(1.24 ; 2.89) \\
\text { OR } 1.74(1.18 ; 2.57) \\
\text { OR } 4.85(1.24 ; 18.96) \\
\text { OR } 6.33(1.65 ; 24.27)\end{array}$ \\
\hline Tedeschi et $\mathrm{al}^{77}$ (2007) & Finland Iceland & $\mathrm{CC}$ & Epstein-Barr virus & ALL & OR $1.9(1.2 ; 3.0)$ \\
\hline \multicolumn{6}{|c|}{ Non-significant effect or no association } \\
\hline Randolph \& Heath ${ }^{59}$ (1974) & USA & $\mathrm{BC}$ & Influenza & $\mathrm{CL}$ & No consistent increased incidence \\
\hline Curnen et al ${ }^{12}(1974)$ & Connecticut & $\mathrm{ECO}$ & $\begin{array}{c}\text { Influenza, chickenpox, } \\
\text { whooping... }\end{array}$ & $\mathrm{CL}$ & $\begin{array}{c}\text { No significant positive } \\
\text { correlations }\end{array}$ \\
\hline Dockerty et $\mathrm{al}^{13}$ (1999) & New Zealand & $\mathrm{CC}$ & $\begin{array}{c}\text { Influenza } \\
\text { Cold sores/oral herpes }\end{array}$ & $\mathrm{CL}$ & $\begin{array}{l}\text { OR } 0.58(0.24 ; 1.41) \\
\text { OR } 0.70(0.25 ; 1.99)\end{array}$ \\
\hline Mckinney et al ${ }^{44}$ (1999) & Scotland & $\mathrm{CC}$ & $\begin{array}{l}\text { Respiratory tract } \\
\text { Genitourinary }\end{array}$ & ALL & $\begin{array}{l}\text { OR } 1.64(0.60 ; 4.46) \\
\text { OR } 1.18(0.50 ; 2.79)\end{array}$ \\
\hline Infante-Rivard et al ${ }^{24}(2000)$ & Quebec & $\mathrm{CC}$ & Recurrent infections & ALL & OR $1.09(0.65 ; 1.84)$ \\
\hline
\end{tabular}

BC: Birth cohort study; CC: Case-control study; ECO: Ecological study; CL: Childhood leukemia; ALL: Acute lymphoblastic leukemia; RR: Relative risk

ALL (especially subtype c-ALL). Whereas limited exposure to infection in this period of life increases the risk of disease by enhancing the possibility of abnormal immune response to infection acquired later.

The observation of temporary CL clusters in relatively isolated communities after increased entry of new individuals into these populations has provided support for the formulation of the "population mixing" hypothesis. Kinlen ${ }^{27,28}$ suggests that the introduction of a specific infectious agent in a non-immune population could cause an abnormal immune response to infection by this pathogen and cause a transient increase in leukemia cases.

This article reviews studies related to Smith and Greaves' hypothesis on the role of infections and indirect measures of infection exposure on the risk of childhood leukemia, especially ALL.

\section{METHODS}

In order to identify eligible studies published before January 3, 2012, a search was made in three scientific publication databases: Medline, ${ }^{a}$ Lilacs $^{\mathrm{b}}$ and SciELO. ${ }^{\mathrm{c}}$ Studies with subjects age $\leq 15$ years old and specific analysis of cases diagnosed as ALL and/or total leukemia were included in this review. The search considered possible associated factors: maternal or infant exposure to infections or proxy of infections (day care attendance, birth order, breastfeeding, vaccination).

The descriptors used for searching in the Medline database were: infection and childhood leukemia. The preliminary list included 612 publications. We pre-selected articles by reading titles and abstracts and created a second list of 145 potentially eligible studies. The inclusion criteria were met in 47 of those 145 articles. The same descriptors were used to search in the SciELO

a PubMed: the bibliographic database [Internet]. Bethesda (MD): National Library of Medicine (US). 1960s. Available from: http://www.ncbi. nlm.nih.gov/pubmed

b LILACS: Literatura Latino-Americana e do Caribe em Ciências da Saúde [Internet]. São Paulo (BR): Bireme/OPS/OMS. 1982. Available from: http://lilacs.bvsalud.org/

c SciELO: Scientific Electronic Library Online [Internet]. São Paulo (BR): Bireme/OPS/FAPESP/CNPq. 1998. Available from: http://www.scielo. org/php/index.php?lang=en 
and Lilacs databases. However, the term infection was replaced by infect\$. The use of "\$" symbol allows recording of words with the same root. The results included eight and fourteen studies, respectively. None met the inclusion criteria.

We looked for studies in the three databases using the descriptors "childhood leukemia", with the addition of words: infection or maternal disease, breastfeeding, day care, vaccination and birth order. We found a further 15 studies that met inclusion criteria and had not previously been screened.

\section{RESULTS}

This review included 62 studies: $85.5 \%$ were case-control, $9.7 \%$ cohort and $4.8 \%$ ecological studies. The studies were conducted in Europe, North America, Asia, Africa, and Oceania and published between 1973 and 2011.

\section{Direct assessment of exposure to infection in the mothers and children}

\section{Maternal infection}

The association between maternal infection during pregnancy and CL was evaluated in 16 studies, and statistically significant increased risks of leukemia were detected in $11^{2,8,21,33,35,36,43,47,77,78,83}$ (Table 1). Associated infections were: influenza, pneumonia, chickenpox, herpes zoster, lower genital tract infection, skin disease, sexually transmitted diseases, Epstein-Barr virus (EBV), and Helicobacter pylori. Laboratory tests were performed for antibodies in maternal serum during pregnancy in three studies. ${ }^{35,36,77}$ Maternal infection with EBV was associated with a significantly increased risk of ALL in two studies (odds ratio [OR] 2.9, 95\% confidence interval $[95 \% \mathrm{CI}] 1.5 ; 5.8$; OR $1.9,95 \% \mathrm{CI}$ $1.2 ; 3.0),{ }^{35,77}$ and Lehtinen et $\mathrm{al}^{36}$ found a high risk of CL (but not ALL) associated with Helicobacter pylori infection. Naumburg et $\mathrm{al}^{47}$ identified a significantly increased risk of both ALL (OR 1.63, 95\%CI 1.04;2.53) and CL (OR 1.78, 95\%CI 1.17;2.72) associated with lower genital tract infections. Likewise, Kwan et $\mathrm{al}^{33}$ showed that the risk of ALL and CL was increased in children whose mothers had influenza/pneumonia and sexually transmitted diseases during the pregnancy. No statistically significant association was found between maternal infection and leukemia in five studies. ${ }^{12,13,24,44,59}$

\section{Childhood infection}

Table 2 shows twenty-three studies that evaluated the association between childhood infection and leukemia. Among those that analyzed infections in the first two years of life, five reported reduced risk of ALL associated with infection in the skin, ${ }^{44}$ ears, ${ }^{48,81}$ or gastrointestinal tract, ${ }^{25}$ and episodes of roseola and/or fever and rash. ${ }^{10}$ Other two studies detected a higher risk of ALL in children with more frequent episodes of upper respiratory tract infection, ${ }^{9,64}$ fungal infection ${ }^{64}$ and chickenpox. ${ }^{9}$ A further five studies found reduced risk associated with some diseases and increased risk associated with others. ${ }^{13,52,66,67,75}$ The association between common cold, fever, history of infection in the infant and leukemia was not significant in two studies. ${ }^{47,82}$

Exposure to infection at any time prior to diagnosis was examined in six studies. ${ }^{25,38,40,43,68,70}$ A protective effect against leukemia in children with herpes labialis, ${ }^{68}$ chickenpox, ${ }^{70}$ and ear infection was detected. ${ }^{38}$ Mckinney et $\mathrm{al}^{43}$ and Jourdan-Da Silva et $\mathrm{al},{ }^{25}$ respectively, identified an increased risk of leukemia correlating with total number of illness and rubella episodes. Macarthur et $\mathrm{al}^{40}$ found a reduced risk of ALL in children with mumps or measles, but the results were not precise.

The occurrence of infection at leukemia diagnosis or in the year before diagnosis was evaluated in seven studies. ${ }^{10,29,41,56,68,70,71}$ Among those, four performed laboratory tests to investigate exposure to specific infections. Petridou et $\mathrm{al}^{56}$ found a lower risk of ALL associated with EBV, human herpes virus 6 (HHV6), and mycoplasma exposure, and increased risk associated with parainfluenza. Other two studies ${ }^{41,71}$ identified increased risk of ALL in children exposed to the herpes simplex virus 1 and 2, hepatitis B virus, and EBV. Schlehofer et $\mathrm{al}^{68}$ found no significant association between EBV infection, HHV6, parvovirus B19, or the adeno-associated virus and ALL.

\section{Indirect assessment of exposure to infections in childhood}

\section{Daycare attendance}

Table 3 presents information on studies that investigated the association between daycare attendance and leukemia. Nine studies identified a statistically significant reduced risk of leukemia related to daycare attendance. ${ }^{15,24-26,37,38,52,54,81}$ Perrilat et $\mathrm{al}^{52}$ and Gilham et $\mathrm{al}^{15}$ detected a more pronounced protective effect for children having started daycare in early life (OR 0.5 , $95 \%$ CI $0.3 ; 1.0$ for age $\leq 6$ months; OR $0.56,95 \% \mathrm{CI}$ $0.37 ; 0.83$ for age $<3$ months) than in those having started daycare at older ages, but the trend for age of starting daycare was not statistically significant. In Jourdan-Da Silva et al, ${ }^{25}$ a statistically significant association was only observed when daycare started before 3 months old (OR 0.6, 95\%CI $0.4 ; 0.8$ ) and the trend was also statistically significant $(\mathrm{p}$ trend $<0.05)$.

In other eight studies no statistically significant association or effects were reported. ${ }^{1,10,48,55,63,65,67,70}$ Neglia et $\mathrm{al}^{48}$ did not find association even when the daycare began before 6 months of age. 
Table 2. Review of studies on childhood infection and risk of leukemia, 1973-2011.

\begin{tabular}{|c|c|c|c|c|c|}
\hline Reference & Place & $\begin{array}{l}\text { Study } \\
\text { design }\end{array}$ & $\begin{array}{c}\text { Infectious agent or } \\
\text { disease }\end{array}$ & Disease & Risk $(95 \% \mathrm{Cl})$ \\
\hline \multicolumn{6}{|c|}{ Infection in the first 2 years of life: protective effect } \\
\hline Dockerty et al ${ }^{13, a}(1999)$ & New Zealand & $\mathrm{CC}$ & Eye infection & ALL & OR $0.2(0.1 ; 0.7)$ \\
\hline Mckinney et al'4 (1999) & Scotland & $\mathrm{CC}$ & Skin infection & $\begin{array}{c}\text { ALL } \\
C L\end{array}$ & $\begin{array}{l}\text { OR } 0.2(0.05 ; 0.87) \\
\text { OR } 0.2(0.05 ; 0.87)\end{array}$ \\
\hline Neglia et $\mathrm{al}^{48}(2000)$ & USA & $\mathrm{CC}$ & Ear infection & ALL & $\begin{array}{l}\text { Ear infection episodes reduced } \\
\text { the risk: } p \text { trend }=0.026\end{array}$ \\
\hline Chan et al ${ }^{10, a}(2002)$ & Hong Kong & $\mathrm{CC}$ & $\begin{array}{c}\text { Roseola and/or fever } \\
+ \text { rash }\end{array}$ & ALL & OR $0.33(0.16 ; 0.68)$ \\
\hline Perrilat et al ${ }^{52, a}(2002)$ & France & $\mathrm{CC}$ & $\begin{array}{l}\text { Surgical procedures: } \\
\text { ear, nose, throat }\end{array}$ & $\mathrm{CL}$ & OR $0.4(0.2 ; 0.9)$ \\
\hline Jourdan-da Silva et al $25, \mathrm{a}(2004)$ & France & $\mathrm{CC}$ & $\begin{array}{l}\geq 4 \text { gastrointestinal } \\
\text { infections }\end{array}$ & ALL & OR $0.1(0.03 ; 0.6)$ \\
\hline Rosembaum et al ${ }^{66, a}(2005)$ & $\begin{array}{l}\text { New York } \\
\text { State }\end{array}$ & $\mathrm{CC}$ & Diarrhoea & ALL & OR $0.69(0.48 ; 0.99)$ \\
\hline Simpson et $a^{75, a}(2007)$ & UK & $\mathrm{CC}$ & Eye infection & ALL & OR $0.7(0.5 ; 0.9)$ \\
\hline Rudant et al ${ }^{67, a}(2010)$ & France & $\mathrm{CC}$ & $\begin{array}{l}\text { Otitis; Bronchiolitis/ } \\
\text { other lower respiratory } \\
\text { tract infections; } \\
\text { Gastroenteritis }\end{array}$ & ALL & $\begin{array}{l}\text { OR } 0.7(0.5 ; 1.0) \\
\text { OR } 0.3(0.2 ; 0.6) \\
\text { OR } 0.3(0.1 ; 0.8)\end{array}$ \\
\hline Urayama et al ${ }^{81}$ (2011) & California & $\mathrm{CC}$ & $\begin{array}{l}\text { Ear infection: non- } \\
\text { Hispanic children; } \\
\text { Hispanic children }\end{array}$ & ALL & $\begin{array}{l}\text { OR } 0.39(0.17 ; 0.91) \\
\text { OR } 0.48(0.27 ; 0.83)\end{array}$ \\
\hline \multicolumn{6}{|c|}{ Infection in the first 2 years of life: increased risk } \\
\hline Dockerty et al ${ }^{13, a}(1999)$ & New Zealand & $\mathrm{CC}$ & Influenza & $\begin{array}{l}\mathrm{CL} \\
\mathrm{ALL}\end{array}$ & $\begin{array}{l}\text { OR } 6.80(1.81 ; 25.66) \\
\text { OR } 6.0(1.4 ; 26.2)\end{array}$ \\
\hline Perrilat et $\mathrm{al}^{52, \mathrm{a}}(2002)$ & France & $\mathrm{CC}$ & Mumps & $\mathrm{CL}$ & OR $3.2(1.1 ; 9.0)$ \\
\hline Rosembaum et al ${ }^{66, a}(2005)$ & $\begin{array}{l}\text { New York } \\
\text { State }\end{array}$ & $\mathrm{CC}$ & $\begin{array}{l}\text { Otitis in the second } \\
\text { year of life }\end{array}$ & $\begin{array}{l}\text { ALL B } \\
\text { lineage }\end{array}$ & OR $1.56(1.02 ; 2.37)$ \\
\hline Roman et al ${ }^{64}(2007)$ & UK & $\mathrm{CC}$ & $\begin{array}{c}\text { Upper respiratory } \\
\text { tract infection Fungal } \\
\text { infection }\end{array}$ & ALL & $\begin{array}{l}\text { OR } 1.3(1.0 ; 1.7) \\
\text { OR } 1.9(1.1 ; 3.2)\end{array}$ \\
\hline Simpson et $\mathrm{al}^{75, a}(2007)$ & UK & $\mathrm{CC}$ & At least one infection & ALL & OR $1.6(1.1 ; 2.2)$ \\
\hline Cardwell et al ${ }^{9}$ (2008) & UK & $\mathrm{CC}$ & $\begin{array}{l}\text { Upper respiratory tract } \\
\text { infections; Chickenpox }\end{array}$ & ALL & $\begin{array}{l}\text { OR } 1.59(1.02 ; 2.49) \\
\text { OR } 2.62(1.12 ; 6.13)\end{array}$ \\
\hline Rudant et al ${ }^{67, a}(2010)$ & France & $\mathrm{CC}$ & $\begin{array}{c}\text { Upper respiratory tract } \\
\text { infections }\end{array}$ & ALL & OR $1.6(1.3 ; 2.0)$ \\
\hline \multicolumn{6}{|c|}{ Infection in the first 2 years of life: non-significant effects or no association } \\
\hline $\begin{array}{l}\text { Van Steensel-Moll et al }{ }^{82} \\
\text { (1986) }\end{array}$ & Netherlands & $\mathrm{CC}$ & Common colds; Fever & ALL & $\begin{aligned} \text { RR 0.8; } & p>0.05 ; \text { RR 0.9; } \\
p & >0.05\end{aligned}$ \\
\hline Naumburg et al ${ }^{47}(2002)$ & Sweden & $\mathrm{CC}$ & $\begin{array}{l}\text { History of infection in } \\
\text { the postpartum }\end{array}$ & $\mathrm{CL}$ & OR $1.0(0.50 ; 2.04)$ \\
\hline \multicolumn{6}{|c|}{ Infection at any time prior to the diagnosis: protective effect } \\
\hline Schlehofer et al68,a (1996) & Germany & $\mathrm{CC}$ & Herpes labialis & $\mathrm{CL}$ & RR $0.38(0.15 ; 0.96)$ \\
\hline Schuz et al70,a (1999) & Germany & $\mathrm{CC}$ & Chickenpox & $\mathrm{AL}$ & OR $0.8(0.7 ; 1.0)$ \\
\hline Ma et $\mathrm{al}^{38} 2(005)$ & California & $\mathrm{CC}$ & $\begin{array}{c}\text { Ear infection (in non- } \\
\text { Hispanic) }\end{array}$ & c-ALL & OR $0.32(0.14 ; 0.74)$ \\
\hline \multicolumn{6}{|c|}{ Infection at any time prior to the diagnosis: increased risk } \\
\hline Mckinney et al ${ }^{43}(1987)$ & UK & $\mathrm{CC}$ & $\begin{array}{c}\text { Number of illness } \\
\text { episodes }\end{array}$ & $\mathrm{CL}$ & RR $1.9(1.0 ; 3.4)$ \\
\hline Jourdan-da Silva et al ${ }^{25, a}(2004)$ & France & $\mathrm{CC}$ & Rubella & ALL & OR $2.4(1.4 ; 4.1)$ \\
\hline \multicolumn{6}{|c|}{ Infection at any time prior to the diagnosis: non-significant effect or no association } \\
\hline Macarthur et al ${ }^{40}(2008)$ & Canada & $\mathrm{CC}$ & $\begin{array}{l}\text { Mumps } \\
\text { Measles } \\
\end{array}$ & ALL & $\begin{array}{l}\text { OR } 0.57(0.13 ; 2.52) \\
\text { OR } 0.62(0.25 ; 1.27)\end{array}$ \\
\hline \multicolumn{6}{|c|}{ Infection at diagnosis or in the year before diagnosis: increased risk } \\
\hline Schuz et al ${ }^{70, a}(1999)$ & Germany & $\mathrm{CC}$ & $\begin{array}{c}\text { Bronchitis } \\
\text { Pneumonia }\end{array}$ & C-ALL & $\begin{array}{l}\text { OR } 1.9(1.3 ; 2.7) \\
\text { OR } 2.6(1.4 ; 4.8)\end{array}$ \\
\hline Chan et $\mathrm{al}^{10, \mathrm{a}}(2002)$ & Hong Kong & $\mathrm{CC}$ & Tonsillitis & C-ALL & OR $2.96(1.32 ; 6.66)$ \\
\hline Kroll et $\mathrm{al}^{29}(2006)$ & Britain & $\mathrm{ECO}$ & Influenza & C-ALL & $\begin{array}{c}\text { Influenza epidemic preceded } \\
\text { peak of c-ALL }\end{array}$ \\
\hline
\end{tabular}




\begin{tabular}{|c|c|c|c|c|c|}
\hline \multicolumn{6}{|c|}{ Infection at diagnosis or in the year before diagnosis: laboratory analysis } \\
\hline Schlehofer et al ${ }^{68, a}(1996)$ & Germany & CC & $\begin{array}{c}\text { Epstein-Barr Virus } \\
\text { Parvovirus B-19 } \\
\text { Adeno-associated virus } \\
\text { type } 2 \\
\text { Human herpes virus } \\
\text { type } 6 .\end{array}$ & $\mathrm{CL}$ & $\begin{array}{l}\text { RR } 2.05(0.99 ; 4.23) \\
\text { RR } 0.48(0.14 ; 1.69) \\
\text { RR } 0.66(0.29 ; 1.50) \\
\text { RR } 1.11(0.54 ; 2.28)\end{array}$ \\
\hline Petridou et al $^{56}(2001)$ & Greece & $\mathrm{CC}$ & $\begin{array}{c}\text { Epstein-Barr virus } \\
\text { Human herpes virus } \\
\text { type } 6 \\
\text { Mycoplasma } \\
\text { Parainfluenza1,2,3 }\end{array}$ & ALL & $\begin{array}{l}\text { OR } 0.4(0.2 ; 0.8) \\
\text { OR } 0.5(0.3 ; 0.9) \\
\text { OR } 0.1(0.0 ; 0.7) \\
\text { OR } 1.9(1.1 ; 3.2)\end{array}$ \\
\hline Mahjour et al ${ }^{41}(2010)$ & Iran & $\mathrm{CC}$ & $\begin{array}{c}\text { Herpes Simplex } \\
\text { Viruses } 1 \text { and 2; } \\
\text { Epstein-Barr Virus; } \\
\text { hepatitis B Virus }\end{array}$ & ALL & $\begin{array}{c}\text { The prevalence of antibodies } \\
\text { against HBsAg }(p=0.002), \\
\text { HSV1 }(p<0.0001), \text { VCA } \\
(p=0.021) \text { and EA }(p<0.0001) \\
\text { antigens of EBV were higher in } \\
\text { ALL patients. }\end{array}$ \\
\hline Sehgal et $\mathrm{al}^{71}(2010)$ & India & $\mathrm{CC}$ & Epstein Barr Virus & ALL & $\begin{array}{l}\text { Significant increase in EBV in ALL } \\
\text { patients }(p<0.05)\end{array}$ \\
\hline
\end{tabular}

CC: Case-control study; ECO: Ecological study; CL: Childhood leukemia; ALL: Acute lymphoblastic leukemia; AL: Acute leukemia; c-ALL: B-cell precursor common AL; RR: Relative risk; OR: Odds ratio; $95 \% \mathrm{Cl}$ : $95 \%$ confidence interval a Study listed more than once in the table

\section{Birth order}

The relationship between birth order and leukemia was evaluated in 21 studies (Table 4). Van SteenselMoll ${ }^{82}$ detected increased ALL risk in children with lower birth order (first-born). Four studies found a protective effect associated with higher birth order. ${ }^{14,56,67,81}$ Of these, Dockerty et al ${ }^{14}$ observed ALL risk reduction with increased parity $(\mathrm{p}$ trend $<0.001$ ) in a sample of 2,942 cases and the same number of controls in England and Wales.

Infante-Rivard et $\mathrm{al}^{24}$ detected a reduced ALL risk in children aged four and over who had at least one older sibling in the first year of life (OR $0.46,95 \% \mathrm{CI}$ $0.22 ; 0.97)$, but they observed a higher ALL risk in children under four years old who had at least one older sibling at diagnosis (OR 4.54, 95\%CI 2.27;9.07).

Three studies found higher ALL risk related to higher birth order. ${ }^{25,61,73}$ Shu et al $^{73}$ in the United States $(1,842$ cases of ALL, 1,986 controls) detected positive association (OR 2.0, 95\% CI 1.3;3.0; $\mathrm{p}$ trend $<0.01$ ). Abdul Rahman et al ${ }^{1}$ identified reduced risk of acute leukemia related to lower birth order.

However, 11 studies showed no statistically significant association. ${ }^{15,26,44,46,48,50,52,55,63,70,85}$ Westergaard et al, ${ }^{85}$ in a cohort study in Denmark, found a reduced risk of ALL related to higher birth order, but the result was not precise (relative risk [RR] 0.72, 95\%CI 0.46;1.13).

\section{Assessment of child immune status}

\section{Breastfeeding}

Table 5 lists the studies that investigated the association between leukemia and breastfeeding.
A protective effect in children with more breastfeeding time was detected in five of those 17 studies. ${ }^{13,24,53,67,72} \mathrm{Shu}$ et $\mathrm{al}^{72}$ identified a reducing ALL risk with increased breastfeeding time ( $\mathrm{p}$ trend $0.003)$. Children who were breastfed for more than six months had a lower risk of ALL (OR 0.72, 95\% CI $0.60 ; 0.87)$ than those who were never breastfed. Two studies found a higher ALL risk in children breastfed for less than six months.

Ten studies found no association between breastfeeding and CL. ${ }^{10,25,32,34,40,46,55,70,79,84}$ In a large study $(1,342 \mathrm{CL}$ cases) conducted in the United Kingdom, no association was seen with breastfeeding duration ( $\mathrm{p}$ trend 0.90). ${ }^{34}$

\section{Vaccination}

Eleven studies explored associations between vaccination and leukemia (data not shown). . $^{3,8,13,20,39,40,42,43,49,55,70}$ Five of these identified a reduced risk in children who were vaccinated (any vaccine) ${ }^{13,43}$ or who had been immunized with $\mathrm{BCG}^{49}$ and Hib (Haemophilus influenzae type B $)^{20,39}$ vaccine. Ma et $\mathrm{al}^{39}$ detected a lower risk of ALL (OR 0.81, 95\%CI 0.66;0.98) and CL (OR 0.81, 95\%CI 0.68;0.96) associated with the Hib vaccine. Furthermore, Schuz et al ${ }^{70}$ observed an increased c-ALL risk in children who had received less than four vaccines compared to those who had received six or more.

On the other hand, Buckley et $\mathrm{al}^{8}$ found an increased risk of c-ALL (OR 1.7, p < 0.01) in children who received the MMR (measles, mumps and rubella) vaccine. Four studies found no statistically significant association between these variables. , $^{3,40,42,55}$ 
Table 3. Review of studies on daycare attendance and risk of childhood leukemia, 1973-2011.

\begin{tabular}{|c|c|c|c|c|c|}
\hline Reference & Place & $\begin{array}{c}\text { Study } \\
\text { design }\end{array}$ & Disease & Variables & Risk $(95 \% \mathrm{Cl})$ \\
\hline \multicolumn{6}{|l|}{ Protective effect } \\
\hline $\begin{array}{l}\text { Petridou et al }{ }^{54} \\
\text { (1993) }\end{array}$ & $\begin{array}{c}\text { Attica } \\
\text { (Greece) }\end{array}$ & $\mathrm{CC}$ & $\mathrm{CL}$ & $\begin{array}{c}\text { Attendance at day-care for }>3 \text { months in the first } \\
\text { two years of life }\end{array}$ & $\begin{array}{l}\text { OR } 0.28 \\
(0.09 ; 0.88)\end{array}$ \\
\hline $\begin{array}{l}\text { Infante-Rivard et a }{ }^{24} \\
(2000)\end{array}$ & Quebec & $\mathrm{CC}$ & ALL & Entry at $\leq 2$ years old & $\begin{array}{c}\text { OR } 0.49 \\
(0.31 ; 0.77)\end{array}$ \\
\hline Perrilat et al ${ }^{52}(2002)$ & France & $\mathrm{CC}$ & $\mathrm{AL}$ & $\begin{array}{c}\text { Age at start of day care } \leq 6 \text { months versus no day- } \\
\text { care }\end{array}$ & $\begin{array}{l}\text { OR } 0.5 \\
(0.3 ; 1.0)\end{array}$ \\
\hline Ma et $\mathrm{al}^{37}(2002)$ & California & $\mathrm{CC}$ & ALL & $\begin{array}{c}\text { Children who had more total child - hours of } \\
\text { attendance at day-care }\end{array}$ & $\begin{array}{c}\text { OR } 0.64 \\
(0.45 ; 0.95)\end{array}$ \\
\hline $\begin{array}{l}\text { Jourdan-Da Silva et } \\
\mathrm{al}^{25}(2004)\end{array}$ & France & $\mathrm{CC}$ & ALL & $\begin{array}{c}\text { Age at start of day-care }<3 \text { months versus no day- } \\
\text { care }\end{array}$ & $\begin{array}{l}\text { OR } 0.6 \\
(0.4 ; 0.8)\end{array}$ \\
\hline Ma et $\mathrm{a}^{38}(2005)$ & California & $\mathrm{CC}$ & ALL & $\begin{array}{c}\text { Children (non-Hispanic white) who had more time } \\
\text { of attendance at day-care }\end{array}$ & $\begin{array}{c}\text { OR } 0.42 \\
(0.18 ; 0.99)\end{array}$ \\
\hline Gilham et al ${ }^{15}$ (2005) & UK & $\mathrm{CC}$ & ALL & $\begin{array}{c}\text { Formal day care in the first year of life } \\
\text { Age at start of day-care }<3 \text { months versus no day- } \\
\text { care }\end{array}$ & $\begin{array}{l}\text { OR } 0.48 \\
(0.37 ; 0.62) \\
\text { OR } 0.56 \\
(0.37 ; 0.83)\end{array}$ \\
\hline $\begin{array}{l}\text { Kamper-Jorgensen et } \\
\mathrm{al}^{26}(2008)\end{array}$ & Denmark & $\mathrm{CC}$ & ALL & Childcare in the first 2 years & $\begin{array}{c}\text { OR } 0.68 \\
(0.48 ; 0.95)\end{array}$ \\
\hline Urayama $^{81}(2011)$ & California & $\mathrm{CC}$ & ALL & $\begin{array}{l}\text { Attendance day-care by age } 6 \text { months (non-Hispanic } \\
\text { white) }\end{array}$ & $\begin{array}{c}\text { OR } 0.83 \\
(0.73 ; 0.94)\end{array}$ \\
\hline \multicolumn{6}{|c|}{ Non-significant effect or no association } \\
\hline Roman et al ${ }^{63}$ (1994) & England & $\mathrm{CC}$ & ALL & Child's attendance at preschool playgroup & $\begin{array}{l}\text { OR } 0.6 \\
(0.2 ; 1.8)\end{array}$ \\
\hline $\begin{array}{l}\text { Petridou et al }{ }^{55} \\
\text { (1997) }\end{array}$ & Greece & $\mathrm{CC}$ & $\mathrm{CL}$ & Day-care: Yes versus No & $\begin{array}{c}\text { OR } 0.83 \\
(0.51 ; 1.37)\end{array}$ \\
\hline Schuz et $\mathrm{al}^{70}(1999)$ & Germany & $\mathrm{CC}$ & $\mathrm{C}-\mathrm{ALL}$ & Deficit in social contact & $\begin{array}{c}\text { OR } 1.0 \\
(0.8 ; 1.2)\end{array}$ \\
\hline Neglia et al ${ }^{48}(2000)$ & USA & $\mathrm{CC}$ & ALL & Age at start of day care $<6$ months & $\begin{array}{c}\text { OR } 0.91 \\
(0.72 ; 1.15)\end{array}$ \\
\hline $\begin{array}{l}\text { Rosenbaum et } \mathrm{al}^{65} \\
(2000)\end{array}$ & $\begin{array}{l}\text { New York } \\
\text { State }\end{array}$ & $\mathrm{CC}$ & ALL & $\begin{array}{l}\text { Duration of out-of-home care (months): stayed home } \\
\qquad \text { (versus }>36)\end{array}$ & $\begin{array}{c}\text { OR } 1.32 \\
(0.70 ; 2.52)\end{array}$ \\
\hline Chan et $\mathrm{al}^{10}(2002)$ & $\begin{array}{l}\text { Hong } \\
\text { Kong }\end{array}$ & $\mathrm{CC}$ & C-ALL & Attendance at day-care during first year of life & $\begin{array}{c}\text { OR } 0.93 \\
(0.63 ; 1.36)\end{array}$ \\
\hline $\begin{array}{l}\text { Abdul Rahman et al }{ }^{1} \\
\text { (2008) }\end{array}$ & Malaysia & $\mathrm{CC}$ & $\mathrm{AL}$ & Attendance in day-care (Yes versus $\mathrm{No}$ ) & $\begin{array}{c}\text { OR } 1.12 \\
(0.65 ; 1.92)\end{array}$ \\
\hline Rudant et $\mathrm{al}^{67}$ (2010) & France & $\mathrm{CC}$ & ALL & Full-time day-care attendance in the first year of life & $\begin{array}{c}\text { OR } 0.8 \\
(0.6 ; 1.1)\end{array}$ \\
\hline
\end{tabular}

CC: Case-control study; CL: Childhood leukemia; ALL: Acute lymphoblastic leukemia; AL: Acute leukemia; c-ALL: B-cell precursor common ALL; OR: Odds ratio; $95 \% \mathrm{Cl}$ : $95 \%$ confidence interval

\section{DISCUSSION}

Greaves $^{17,19}$ argues that infections play an important role in the natural history of ALL, and especially c-ALL, considering the two hit model involving two independent genetic mutations. The first event concerns the initial genetic damage which occurs in the uterus during B cell precursor expansion producing a pre-leukemic clone. The second concerns postnatal genetic mutation that can lead to disease development.

According to this review, more than $50.0 \%$ of studies investigating maternal exposure to infection observed increasing risk of $\mathrm{CL}$. These results support the hypothesis proposed by Smith. ${ }^{76}$ It is possible that exposure to in utero infection is one of the factors involved in genetic damage in the first "hit" referred to by Greaves. ${ }^{17}$

Results of childhood exposure to infections support the hypothesis that infection early in life (especially the first year of life) is associated with reduced risk of ALL. Even so, data are conflicting. Some factors may contribute to the apparent inconsistency between studies. ${ }^{\mathrm{d}}$ First, infections may only be involved in the etiology of a specific subtype of ALL (most probably c-ALL). For this reason, recent studies have analyzed all cases of ALL and specifically c-ALL. Another difficulty concerns when the infections occurred. Studies evaluating infection occurrence

${ }^{d}$ Edgar K, Morgan A. Does infections cause or prevent childhood leukemia? A review of the scientific evidence. London: Children with Leukemia; 2008 [cited 2013 Oct 31]. Available from: http://www.childrenwithcancer.org.uk/infection-and-leukaemia 
Table 4. Review of studies on birth order and risk of childhood leukemia, 1973-2011.

\begin{tabular}{|c|c|c|c|c|c|}
\hline Reference & Place & $\begin{array}{l}\text { Study } \\
\text { design }\end{array}$ & Disease & Variables & Risk $(95 \% \mathrm{Cl})$ \\
\hline \multicolumn{6}{|c|}{ Negative association: protective effect associated with higher birth order/ increased risk associated with lower birth order } \\
\hline $\begin{array}{l}\text { Van steensel-moll }{ }^{82} \\
(1986)\end{array}$ & Netherlands & $\mathrm{CC}$ & ALL & There were more first-born children in cases & RR $1.8(1.1 ; 2.7)$ \\
\hline $\begin{array}{l}\text { Infante-Rivard et } \\
\mathrm{al}^{24, a}(2000)\end{array}$ & Quebec & $\mathrm{CC}$ & ALL & $\begin{array}{l}\text { Having older siblings in the } 1^{\text {st }} \text { year of life (in } \\
\text { cases diagnosed at } 4 \text { years of age or later) }\end{array}$ & $\begin{array}{l}\text { OR } 0.46 \\
(0.22 ; 0.97)\end{array}$ \\
\hline $\begin{array}{l}\text { Petridou et al }{ }^{56} \\
\text { (2001) }\end{array}$ & Greece & $\mathrm{CC}$ & ALL & Birth order: other versus first & OR $0.5(0.3 ; 0.9)$ \\
\hline $\begin{array}{l}\text { Dockerty et al }{ }^{14} \\
\text { (2001) }\end{array}$ & $\begin{array}{l}\text { England } \\
\text { Wales }\end{array}$ & $\mathrm{CC}$ & ALL & Parity $\geq 5$ & $\begin{array}{l}\text { OR } 0.52 \\
(0.34 ; 0.80)\end{array}$ \\
\hline $\begin{array}{l}\text { Rudant et al }{ }^{67} \\
(2010)\end{array}$ & France & $\mathrm{CC}$ & ALL & Parity $\geq 4$ versus 1 & OR $0.5(0.3 ; 0.8)$ \\
\hline Urayama $^{81}(2011)$ & California & $\mathrm{CC}$ & ALL & $\begin{array}{c}\text { Birth order } \geq 4 \text { versus } 1 \text { (in children non- } \\
\text { Hispanic white) }\end{array}$ & $\begin{array}{c}\text { OR } 0.44 \\
(0.21 ; 0.92)\end{array}$ \\
\hline \multicolumn{6}{|c|}{ Positive association: increased risk associated with higher birth order/ protective effect associated with lower birth order } \\
\hline $\begin{array}{l}\text { Infante-Rivard et } \\
\mathrm{al}^{24, \mathrm{a}}(2000)\end{array}$ & Quebec & $\mathrm{CC}$ & ALL & $\begin{array}{l}\text { Having older siblings at time of diagnosis (in } \\
\text { children diagnosed before } 4 \text { years of age) }\end{array}$ & $\begin{array}{c}\text { OR } 4.54 \\
(2.27 ; 9.07)\end{array}$ \\
\hline Shu et $\mathrm{al}^{73}(2002)$ & USA & $\mathrm{CC}$ & ALL & Birth order $\geq 4$ versus 1 & OR $2.0(1.3 ; 3.0)$ \\
\hline $\begin{array}{l}\text { Reynolds et al }{ }^{61} \\
(2002)\end{array}$ & California & $\mathrm{CC}$ & ALL & $\begin{array}{l}\text { Number of previous live births } \geq 3 \text { versus } 0 \\
\text { (in children aged }<2 \text { years) }\end{array}$ & $\begin{array}{c}\text { OR } 1.53 \\
(1.00 ; 2.34)\end{array}$ \\
\hline $\begin{array}{l}\text { Jourdan-Da Silva et } \\
\mathrm{al}^{25}(2004)\end{array}$ & France & $\mathrm{CC}$ & ALL & Birth order $\geq 4$ versus 1 & OR $2.0(1.1 ; 3.7)$ \\
\hline $\begin{array}{l}\text { Abdul Rahman et } \\
\mathrm{al}^{1}(2008)\end{array}$ & Malaysia & $\mathrm{CC}$ & $\mathrm{AL}$ & Number of elder siblings $<2$ versus $\geq 2$ & $\begin{array}{c}\text { OR } 0.37 \\
(0.22 ; 0.64)\end{array}$ \\
\hline \multicolumn{6}{|c|}{ Non-significant effect or no association } \\
\hline $\begin{array}{l}\text { Roman et al63 } \\
(1994)\end{array}$ & England & $\mathrm{CC}$ & ALL & Number of siblings $\geq 2$ versus 0 & OR $0.8(0.2 ; 3.0)$ \\
\hline $\begin{array}{l}\text { Westergaard et a }{ }^{85} \\
\text { (1997) }\end{array}$ & Denmark & $\mathrm{COH}$ & ALL & Birth order $\geq 4$ versus 1 & $\begin{array}{c}\text { RR } 0.72 \\
(0.46 ; 1.13)\end{array}$ \\
\hline $\begin{array}{l}\text { Petridou et al }{ }^{55} \\
\text { (1997) }\end{array}$ & Greece & $\mathrm{CC}$ & $\mathrm{CL}$ & Risk decreases with increasing birth order & $\begin{array}{c}\text { OR } 0.74 \\
(0.48 ; 1.15)\end{array}$ \\
\hline $\begin{array}{l}\text { Schuz et al }{ }^{70} \\
(1999)\end{array}$ & German & $\mathrm{CC}$ & C-ALL & First-born child (yes versus no) & OR $1.1(1.0 ; 1.4)$ \\
\hline $\begin{array}{l}\text { Mckinney et } \mathrm{al}^{44} \\
\text { (1999) }\end{array}$ & Scotland & $\mathrm{CC}$ & $\begin{array}{l}\mathrm{ALL} \\
\mathrm{CL}\end{array}$ & Parity (0 versus 1 or more) & $\begin{array}{c}\text { OR } 0.81 \\
(0.52 ; 1.25) \\
\text { OR } 0.82 \\
(0.55 ; 1.23)\end{array}$ \\
\hline $\begin{array}{l}\text { Neglia et al }{ }^{48} \\
(2000)\end{array}$ & USA & $\mathrm{CC}$ & ALL & Number of older siblings $\geq 2$ versus 0 & $\begin{array}{c}\text { OR } 1.05 \\
(0.88 ; 1.26)\end{array}$ \\
\hline $\begin{array}{l}\text { Perrilat et a }{ }^{52} \\
(2002)\end{array}$ & France & $\mathrm{CC}$ & ALL & Birth order $\geq 4$ versus 1 & OR $1.4(0.7 ; 2.8)$ \\
\hline $\begin{array}{l}\text { Murray et al }{ }^{46} \\
(2002)\end{array}$ & Ireland & $\mathrm{COH}$ & ALL & First-born versus Not first born & $\begin{array}{c}\text { RR } 0.98 \\
(0.71 ; 1.36)\end{array}$ \\
\hline Okcu et $a^{50}(2002)$ & Texas & $\mathrm{CC}$ & $\begin{array}{c}\text { ALL } \\
\text { CL }\end{array}$ & Parity $\geq 5$ versus 0 & $\begin{array}{l}\text { OR } 1.0(0.1 ; 7.8) \\
\text { OR } 0.5(0.1 ; 4.3)\end{array}$ \\
\hline $\begin{array}{l}\text { Gilham et al }{ }^{15} \\
(2005)\end{array}$ & UK & $\mathrm{CC}$ & ALL & Number of siblings $\geq 3$ versus none & $\begin{array}{c}\text { OR } 0.99 \\
(0.74 ; 1.30)\end{array}$ \\
\hline $\begin{array}{l}\text { Kamper-Jorgensen } \\
\text { et } \mathrm{a}^{26}(2008)\end{array}$ & Denmark & $\mathrm{CC}$ & ALL & Older siblings $>2$ versus 0 & $\begin{array}{c}\text { RR } 0.93(0.72 ; \\
1.19)\end{array}$ \\
\hline
\end{tabular}

CC: Case-control study; COH: Cohort study; CL: Childhood leukemia; ALL: Acute lymphoblastic leukemia; AL: Acute leukemia; c-ALL: B-cell precursor common ALL; RR: Relative risk; OR: Odds ratio; 95\%Cl: 95\% confidence interval a Study listed more than once in the table

at any time prior to diagnosis showed more inconsistent results. Future studies should evaluate the infection occurrence in specific periods such as the first year and one year before diagnosis. In this way, data would help assess whether a lack of immune system modulation in early childhood, associated with a delay in infection occurrence, increases the risk of ALL. In relation to the display window, infections detected near the leukemia diagnosis may be due to an increased susceptibility to infections resulting from the effects of leukemia itself. Some protocols exclude data on infections occurring in the three months before diagnosis date to avoid bias. 
Table 5. Review of studies on breastfeeding and risk of childhood leukemia, 1973-2011.

\begin{tabular}{|c|c|c|c|c|c|}
\hline Reference & Place or group & $\begin{array}{l}\text { Study } \\
\text { design }\end{array}$ & Disease & Time of breast feeding & Risk $(95 \% \mathrm{Cl})$ \\
\hline \multicolumn{6}{|c|}{$\begin{array}{l}\text { Negative association: protective effect associated with longer breastfeeding period/increased risk associated with shorter } \\
\text { breastfeeding period }\end{array}$} \\
\hline Dockerty et al ${ }^{13}(1999)$ & New Zealand & $\mathrm{CC}$ & ALL & $>1$ year & OR $0.5(p=0.04)$ \\
\hline Shu et $\mathrm{al}^{72}(1999)$ & $\begin{array}{l}\text { USA, Canada, } \\
\text { Australia }\end{array}$ & $\mathrm{CC}$ & ALL & $\begin{array}{l}>6 \text { versus } 0 \\
\text { More time breastfeeding }\end{array}$ & $\begin{array}{l}\text { OR } 0.72(0.60 ; 0.87) \\
\quad p \text { trend }=0.0034\end{array}$ \\
\hline Infante-Rivard et al ${ }^{24}(2000)$ & Quebec & $\mathrm{CC}$ & ALL & $>3$ months versus 0 & OR $0.67(0.47 ; 0.94)$ \\
\hline Bener et $\mathrm{al}^{6}(2001)$ & $\begin{array}{l}\text { United Arab } \\
\text { Emirates }\end{array}$ & $\mathrm{CC}$ & ALL & $0-6$ months versus $>6$ & OR $2.47(1.17 ; 5.25)$ \\
\hline Perrilat et al ${ }^{53}(2002)$ & France & $\mathrm{CC}$ & $\mathrm{AL}$ & $\geq 6$ months versus 0 & OR $0.5(0.2 ; 0.9)$ \\
\hline Bener et $\mathrm{al}^{7}(2008)$ & Qatar & $\mathrm{CC}$ & ALL & $0-6$ months versus $>6$ & $\begin{array}{l}\text { Males: OR } 3.1(1.4 ; 6.8) \\
\text { Females: OR } 2.2(0.8 ; 6.32)\end{array}$ \\
\hline Rudant et al $^{67}(2010)$ & France & $\mathrm{CC}$ & ALL & $\geq 6$ months versus $>0$ & OR $0.7(0.5 ; 1.0)$ \\
\hline \multicolumn{6}{|c|}{ Non-significant effect or no association } \\
\hline Petridou et al ${ }^{55}$ (1997) & Greece & $\mathrm{CC}$ & $\mathrm{CL}$ & yes versus no & OR $0.85(0.52 ; 1.41)$ \\
\hline Schuz et al ${ }^{70}$ (1999) & Germany & $\mathrm{CC}$ & C-ALL & $2-6$ months versus $>6$ & OR $1.2(0.9 ; 1.6)$ \\
\hline $\operatorname{UKCCS}^{79}(2001)$ & UK & $\mathrm{CC}$ & ALL & $\geq 7$ months versus 0 & OR $0.89(0.75 ; 1.05)$ \\
\hline Murray et al ${ }^{46}(2002)$ & Ireland & $\mathrm{COH}$ & ALL & no versus yes & RR $0.98(0.68 ; 1.42)$ \\
\hline Chan et $\mathrm{al}^{10}(2002)$ & Hong Kong & $\mathrm{CC}$ & C-ALL & $\begin{array}{l}\geq 6 \text { months (yes versus } \\
\text { no) }\end{array}$ & OR $0.21(0.03 ; 1.76)$ \\
\hline $\begin{array}{l}\text { Lancashire \& Sorahan }{ }^{34} \\
(2003)\end{array}$ & UK & $\mathrm{CC}$ & $\begin{array}{c}\mathrm{ALL} \\
\mathrm{CL}\end{array}$ & ever versus never & $\begin{array}{l}\text { OR } 1.04(0.86 ; 1.26) \\
\text { OR } 1.05(0.89 ; 1.23)\end{array}$ \\
\hline $\begin{array}{l}\text { Jourdan-Da Silva et al }{ }^{25} \\
(2004)\end{array}$ & France & $\mathrm{CC}$ & ALL & yes versus no & OR $1.1(0.9 ; 1.5)$ \\
\hline Kwan et al ${ }^{32}(2005)$ & California & $\mathrm{CC}$ & ALL & ever versus never & OR $0.99(0.64 ; 1.55)$ \\
\hline Macarthur et $\mathrm{al}^{40}(2008)$ & Canada & $\mathrm{CC}$ & $\begin{array}{c}\mathrm{ALL} \\
\mathrm{CL}\end{array}$ & $\begin{array}{c}\text { 7-12 months (yes versus } \\
\text { no) }\end{array}$ & $\begin{array}{l}\text { OR } 1.02(0.68 ; 1.53) \\
\text { OR } 1.00(0.67 ; 1.50)\end{array}$ \\
\hline Waly et al ${ }^{84}$ (2011) & Oman & $\mathrm{CC}$ & ALL & $\begin{array}{c}\text { No statistically } \\
\text { significant difference }\end{array}$ & $\chi 2=3.816, P=0.282$ \\
\hline
\end{tabular}

CC: Case-control study; COH: Cohort study; CL: Childhood leukemia; ALL: Acute lymphoblastic leukemia; AL: Acute leukemia; c-ALL: B-cell precursor common ALL; RR: Risk relative; OR: Odds ratio; $95 \% \mathrm{Cl}$ : 95\% confidence interval

The instrument used to directly assess exposure to infection is another limitation we found. Most studies used data from mothers via questionnaires for the recall of infection occurrence. This method is subject to misclassification bias, with differential recall between cases and controls. Only three of the studies in this review obtained clinical records to evaluate children's exposure to infection. ${ }^{9,64,75}$ Although this latter method is not affected by recall bias, it does present other difficulties. The lack of a clinical records system with extensive population coverage and good quality data hinders the use of this instrument. Furthermore, the number of infections can be underestimated because the occurrence of common infections does not always mean that the mother seeks health service help. Simpson et a $7^{75}$ compared results from clinical diagnoses of infection with those based on maternal self-report. They observed that mothers of cases and controls under-reported the frequency of infections in the first year of life. However, the degree of under-reporting appeared greater for mothers of cases than of controls.

Chang et $\mathrm{al}^{11}$ used records of medically diagnosed infections and they found higher risk of ALL in children who had had acute respiratory infections and any infections before 1 year of age. The authors suggest that children who develop leukemia may have dysregulated immune function since early childhood strongly reacting to infections.

However, they do not refute the "delayed infection hypothesis" supported by several studies using proxy measures and state that their results are not relevant to asymptomatic infections or infections not requiring medical attention.

The difficulty in measuring the occurrence of infectious diseases in childhood leads epidemiologists to use proxy measures of exposure to infection: indirect measures that indicate levels of physical and social contact. These variables are easier to obtain, are less prone to recall bias, and probably include children with asymptomatic infections.

Daycare attendance has been widely used as an indicator of a child's level of social contact and therefore opportunity of contact with infectious agents. Interaction between children, parents, and staff, and the sharing of toys are factors that may contribute to increased transmission of infectious agents. Exposure to respiratory and gastrointestinal tracts infections are known to be more frequent in this type of environment. ${ }^{23,51}$ In general, the studies in our review show 
evidence supporting a reduced risk of ALL associated with daycare attendance, providing support to the hypothesis proposed by Greaves. In a meta-analysis, Urayama et $\mathrm{al}^{80}$ found a reduced risk of ALL in two subgroups of children - those who attended day-care before two years of age and those where age at day-care attendance was not specified (any age before diagnosis).

Birth order has also been used as a proxy variable for infection since children with older siblings are more likely to be exposed to infection due to contact with their siblings. ${ }^{\mathrm{d}}$ Most studies showed no evidence of an association between these variables. Parity is probably influenced by selection bias. Couples with higher socioeconomic status (SES) tend to have fewer children so there may be some confounding effect if SES is assessed differently between groups (cases and controls). Moreover, Edgar \& Morgan ${ }^{\mathrm{d}}$ report that studies generally do not differentiate between the firstborn and the only child. Then, the association of ALL with a history of abortion and reproductive failures may be a confounding factor.

The association between SES and CL has been discussed in the literature. SES does influence the level of exposure and vulnerability of individuals to certain diseases. However, some studies have shown that increased risk of CL seems to be associated with increased SES. ${ }^{30,62}$ In Sao Paulo, Southwestern Brazil, Ribeiro et $\mathrm{al}^{62}$ found a lower risk of leukemia in children living in areas with lower SES and in areas where a high percentage of families had more than seven members. However, in the review conducted by Poole et al, ${ }^{58}$ the association direction seems to vary according to study design, place and time; they also highlighted comparison difficulties between studies because of differences in assessing SES. Measures such as family income and parental education presented an inverse association. Evaluation considering professional class revealed increased CL risk associated with higher SES in both ecological and individual approach studies.

Breast milk protects the child against infections and boosts the immune system contributing to its modulation. $^{22}$ It is therefore reasonable to assume that breastfeeding exerts a similar effect to early infection on a child's immunity inducing a protective effect against leukemia. ${ }^{\mathrm{d}}$ Most of the studies in our review did not identify a statistically significant association between ALL and breastfeeding duration. However, in a meta-analysis including 14 studies, Kwan et $\mathrm{al}^{31}$ identified a protective effect from breastfeeding on ALL.

While not an actual infection, vaccine acts on the immune system as an infection does. ${ }^{\mathrm{d}}$ Results from the reviewed studies have provided some evidence of a protective effect from vaccination on the risk of ALL, especially regarding the Hib vaccine. One major difficulty with this variable is that high vaccination coverage makes it difficult to gauge the effect on CL. ${ }^{\mathrm{d}}$

Although no specific etiological agent has been identified, results of the studies reviewed provide evidence that the lack of exposure to infections during early childhood, and consequent failure in modulation of the immune system, may increase the risk of developing leukemia by the occurrence of an abnormal immune response after exposure to a later infection, as reported by Greaves. ${ }^{17,18}$ However, the mechanisms involved in this process are still not completely clear.

Schmiegelow et $\mathrm{al}^{69}$ proposed in 2008 an explanation for the observed association between infections in early childhood and reduced risk of ALL: the adrenal hypothesis. This postulates that children with lower SES, thus subject to getting infections more often, have a lower risk of ALL, because changes in the hypothalamic-pituitary-adrenal axis induced by infection lead to increased cortisol plasma levels similar to those observed during antileukemic therapy, leading to pre-leukemic cell apoptosis. Furthermore, the immune system can adapt to high infectious loads preventing a more reactive inflammatory response induced by Th1 cytokines in that cortisol favors the production of anti-inflammatory Th2 cytokines. Azevedo-Silva et $\mathrm{al}^{4}$ show differences in ALL incidence rates across Brazilian regions. Salvador and Aracaju, cities in the Northeast, had the lowest rates, while Curitiba, Southeast, and Goiania, Midwest, had the highest. Brazilian regions show very distinct social and economic profiles. These authors suggest that the differences in incidence rates could be due to an early exposure to infectious agents in children living in areas with characteristics favoring the continued exposure to such agents. The lower ALL incidence rates in children from those cities (less developed) would then support the adrenal hypothesis.

Besides the lack of immune system modulation, WIEMELS ${ }^{86}$ reports another phenomenon that would leads to an increased risk of CL: children with dysregulated immune function at birth are at higher risk for developing leukemia due to constitutively lower expression of IL-10, a cytokine that is critical in preventing an overactive inflammatory response to pathogenic infections. This factor would explain why in some studies ${ }^{11}$ children with ALL had significantly more clinically diagnosed infectious episodes in the first year of life compared to controls.

The purpose of this study was to review epidemiological studies related to two hypotheses which propose a role for infections in CL etiology. Considering the "two hit" model by Greaves, ${ }^{17}$ it is possible that the Smith hypothesis may play a role in the first hit and delayed infection in the second. Overall, this review provides evidence supporting the role of infections in the natural history of CL. This justifies further research even though no specific infectious agent has so far been associated with 
the disease. With regard to assessing exposure to infection or modulation of the immune system using a proxy, results were more consistent using daycare attendance than birth order, breastfeeding, or vaccination. Future studies should consider carefully evaluating exposure to infection at different stages of childhood. Accurate data on exposure period (in early childhood or near diagnosis), especially in relation to daycare attendance and the occurrence of infection episodes, are essential in eliminating potential bias and improving study accuracy. Furthermore, different subtypes of the disease require specific analysis because infectious agents can have distinct roles in the etiology of each subtype. Specific attention to these points, in addition to the contribution from genetic studies, is essential to help clarify the relationship between infections and CL etiology.

\section{REFERENCES}

1. Abdul Rahman HI, Shah SA, Alias H, Ibrahim HM. A case-control study on the association between environmental factors and the occurrence of acute leukemia among children in Klang Valley, Malaysia. Asian Pac J Cancer Prev. 2008;9(4):649-652.

2. Austin DF, Karp S, Dworsky R, Henderson BE. Excess leukemia in cohorts of children born following influenza epidemics. Am J Epidemiol. 1975;101(1):77-83.

3. Auvinen A, Hakulinen T, Groves F. Haemophilus influenzae type $B$ vaccination and risk of childhood leukaemia in a vaccine trial in Finland. $\mathrm{Br} /$ Cancer. 2000;83(7):956-8. DOI:10.1054/bjoc.2000.1382

4. Azevedo-Silva F, Camargo B, Pombo-deOliveira MS. Implications of infectious diseases and the adrenal hypothesis for the etiology of childhood acute lymphoblastic leukemia. Braz I Med Biol Res. 2010;43(3):226-9. DOI:10.1590/S0100-879X2010007500011

5. Belson M, Kingsley B, Holmes A. Risk factors for acute leukemia in children: a review. Environ Health Perspect. 2007;115(1):138-45. DOI:10.1289/ehp.9023

6. Bener A, Denic S, Galadari S. Longer breast-feeding and protection against childhood leukaemia and lymphomas. Eur J Cancer. 2001;37(2):234-8. DOI:10.1016/S0959-8049(00)00339-7

7. Bener A, Hoffmann GF, Afify Z, Rasul K, Tewfik I. Does prolonged breastfeeding reduce the risk for childhood leukemia and lymphomas? Minerva Pediatr. 2008;60(2):155-61.

8. Buckley JD, Buckley CM, Ruccione K, Sather HN, Waskerwitz MJ, Woods WG, et al. Epidemiological characteristics of childhood acute lymphocytic leukemia: analysis by immunophenotype. The Childrens Cancer Group. Leukemia. 1994;8(5):856-64.

9. Cardwell CR, McKinney PA, Patterson CC, Murray LJ. Infections in early life and childhood leukaemia risk: a UK case-control study of general practitioner records. Br J Cancer. 2008;99(9):1529-33. DOI:10.1038/sj.bjc.6604696

10. Chan LC, Lam TH, Li CK, Lau YL, Li CK, Yuen HL, et al. Is the timing of exposure to infection a major determinant of acute lymphoblastic leukaemia in Hong Kong? Paediatr Perinat Epidemiol. 2002;16(2):154-65. DOI:10.1046/j.1365-3016.2002.00406.x

11. Chang JS, Tsai CR, Tsai YW, Wiemels JL. Medically diagnosed infections and risk of childhood leukaemia: a population-based case-control study. Int J Epidemiol. 2012:41(4):1050-9. DOI:10.1093/ije/dys113

12. Curnen MG, Varma AA, Christine BW, Turgeon LR. Childhood leukemia and maternal infectious diseases during pregnancy. J Natl Cancer Inst. 1974;53(4):943-7.

13. Dockerty JD, Skegg DC, Elwood JM, Herbison GP, Becroft DM, Lewis ME. Infections, vaccinations, and the risk of childhood leukaemia. Br J Cancer. 1999;80(9):1483-9. DOI:10.1038/sj.bjc.6690548

14. Dockerty JD, Draper G, Vincent T, Rowan SD, Bunch KJ. Case-control study of parental age, parity and socioeconomic level in relation to childhood cancers. Int J Epidemiol. 2001;30(6):1428-37. DOI:10.1093/ije/30.6.1428

15. Gilham C, Peto J, Simpson J, Roman E, Eden TO, Greaves MF, et al. Day care in infancy and risk of childhood acute lymphoblastic leukaemia: findings from UK case-control study. BMJ. 2005;330(7503):1294. DOI:10.1136/bmj.38428.521042.8F

16. Greaves M. Molecular genetics, natural history and the demise of childhood leukaemia. Eur J Cancer. 1999;35(2):17385. DOI:10.1016/S0959-8049(98)00433-X

17. Greaves M. Childhood leukaemia. BMJ. 2002;324(7332):283-7. DOI:10.1136/bmj.324.7332.283

18. Greaves M. Infection, immune responses and the aetiology of childhood leukaemia. Nat Rev Cancer. 2006;6(3):193-203. DOI:10.1038/nrc1816

19. Greaves MF. Speculations on the cause of childhood acute lymphoblastic leukemia. Leukemia. 1988;2(2):120-5.

20. Groves FD, Gridley G, Wacholder S, Shu XO, Robison LL, Neglia JP, et al. Infant vaccinations and risk of childhood acute lymphoblastic leukaemia in the USA. Br J Cancer. 1999;81(1):175-8. DOI: $10.1038 /$ sj.bjc.6690668

21. Hakulinen $T$, Hovi $L$, Karkinen-Jääskeläinen $M$, Penttinen K, Saxén L. Association between influenza during pregnancy and childhood leukaemia. Br Med J. 1973;4(5887):265-7.

22. Hanson LA. Breastfeeding provides passive and likely long-lasting active immunity. Ann Allergy Asthma Immunol. 1998;81(6):523-37. DOI:10.1016/S1081-1206(10)62704-4

23. Holmes SJ, Morrow AL, Pickering LK. Child-care practices: effects of social change on the epidemiology of infectious diseases and antibiotic resistance. Epidemiol Rev. 1996;18(1):10-28. 
24. Infante-Rivard C, Fortier I, Olson E. Markers of infection, breast-feeding and childhood acute lymphoblastic leukaemia. Br J Cancer. 2000;83(11):1559-64. DOI:10.1054/bjoc.2000.1495

25. Jourdan-Da Silva N, Perel Y, Méchinaud F, Plouvier E, Gandemer V, Lutz P, et al. Infectious diseases in the first year of life, perinatal characteristics and childhood acute leukaemia. Br J Cancer. 2004;90(1):139-45. DOI:10.1038/sj.bjc.6601384

26. Kamper-Jørgensen M, Woodward A, Wohlfahrt J, Benn $\mathrm{CS}$, Simonsen J, Hjalgrim $\mathrm{H}$, et al. Childcare in the first 2 years of life reduces the risk of childhood acute lymphoblastic leukemia. Leukemia. 2008;22(1):189-93. DOI:10.1038/sj.leu.2404884

27. Kinlen L. Evidence for an infective cause of childhood leukaemia: comparison of a Scottish new town with nuclear reprocessing sites in Britain. Lancet. 1988;2(8624):1323-7. DOI: 10.1016/S0140-6736(88)90867-7

28. Kinlen L, Balkwill A. Infective cause of childhood leukaemia and wartime population mixing in Orkney and Shetland, UK. Lancet. 2001;357(9259):858. DOI:10.1016/S0140-6736(00)04208-2

29. Kroll ME, Draper GJ, Stiller CA, Murphy MFG. Childhood Leukemia Incidence in Britain, 19742000: time trends and possible relation to influenza epidemics. J Nat/ Cancer Inst. 2006;98(6):417-20. DOI:10.1093/jnci/djj095

30. Kroll ME, Stiller CA, Murphy MF, Carpenter LM. Childhood leukaemia and socioeconomic status in England and Wales 1976-2005: evidence of higher incidence in relatively affluent communities persists over time. Br J Cancer. 2011;105(11):1783-7. DOI:10.1038/bjc.2011.415

31. Kwan ML, Buffler PA, Abrams B, Kiley VA. Breastfeeding and the risk of childhood leukemia: a metaanalysis. Public Health Rep. 2004;119(6):521-35. DOI:10.1016/j.phr.2004.09.002

32. Kwan ML, Buffler PA, Wiemels JL, Metayer C, Selvin $\mathrm{S}$, Ducore JM, et al. Breastfeeding patterns and risk of childhood acute lymphoblastic leukaemia. $\mathrm{Br} /$ Cancer. 2005;93(3):379-84. DOI:10.1038/sj.bjc.6602706

33. Kwan ML, Metayer C, Crouse V, Buffler PA. Maternal illness and drug/medication use during the period surrounding pregnancy and risk of childhood leukemia among offspring. Am / Epidemiol. 2007;165(1):27-35. DOI:10.1093/aje/kwj336

34. Lancashire RJ, Sorahan T; OSCC. Breastfeeding and childhood cancer risks: OSCC data. $\mathrm{Br} J$ Cancer. 2003;88(7):1035-7. DOI:10.1038/sj.bjc.6600881

35. Lehtinen M, Koskela P, Ogmundsdottir HM, Bloigu A, Dillner J, Gudnadottir $M$, et al. Maternal herpesvirus infections and risk of acute lymphoblastic leukemia in the offspring. Am J Epidemiol. 2003;158(3):207-13. DOI:10.1093/aje/kwg137

36. Lehtinen M, Ogmundsdottir HM, Bloigu A, Hakulinen T, Hemminki E, Gudnadottir M, et al. Associations between three types of maternal bacterial infection and risk of leukemia in the offspring. Am J Epidemiol. 2005;162(7):662-7. DOI:10.1093/aje/kwi261
37. Ma X, Buffler PA, Selvin S, Matthay KK, Wiencke JK, Wiemels JL, et al. Day-care attendance and risk of childhood acute lymphoblastic leukaemia. Br J Cancer. 2002;86(9):1419-24. DOI:10.1038/sj.bjc.6600274

38. Ma X, Buffler PA, Wiemels JL, Selvin S, Metayer C, Loh M, et al. Ethnic difference in daycare attendance, early infections, and risk of childhood acute lymphoblastic leukemia. Cancer Epidemiol Biomarkers Prev. 2005;14(8):1928-34. DOI:10.1158/1055-9965.EPI-05-0115

39. Ma X, Does MB, Metayer C, Russo C, Wong A, Buffler PA. Vaccination history and risk of childhood leukaemia. Int J Epidemiol. 2005;34(5):1100-9. DOI:10.1093/ije/dyi113

40. MacArthur AC, McBride ML, Spinelli JJ, Tamaro S, Gallagher RP, Theriault GP. Risk of childhood leukemia associated with vaccination, infection, and medication use in childhood: the Cross-Canada Childhood Leukemia Study. Am J Epidemiol. 2008;167(5):598606. DOI:10.1093/aje/kwm339

41. Mahjour SB, Ghaffarpasand F, Fattahi MJ, Ghaderi A, Fotouhi Ghiam A, Karimi M. Seroprevalence of human herpes simplex, hepatitis B and epstein-barr viruses in children with acute lymphoblastic leukemia in southern Iran. Pathol Oncol Res. 2010;16(4):579-82. DOI:10.1007/s12253-010-9258-6

42. Mallol-Mesnard F, Menegaux A, Auvrignon A, Auclerc MF, Bertrand Y, Nelken B, et al. Vaccination and the risk of childhood acute leukaemia: the ESCALE study (SFCE). Int / Epidemiol. 2007;36(1):110-6. DOI:10.1093/ije/dyl270

43. McKinney PA, Cartwright RA, Saiu JM, Mann JR, Stiller CA, Draper GJ, et al. The Inter-Regional Epidemiological Study of Childhood Cancer (IRESCC): a case control study of aetiological factors in leukaemia and lymphoma. Arch Dis Child. 1987;62(3):279-87.

44. McKinney PA, Juszczak E, Findlay E, Smith K, Thomson CS. Pre- and perinatal risk factors for childhood leukaemia and other malignancies: a Scottish case control study. Br / Cancer. 1999;80(11):1844-51. DOI:10.1038/sj.bjc.6690609

45. Mori H, Colman SM, Xiao Z, Ford AM, Healy LE, Donaldson C, et al. Chromosome translocations and covert leukemic clones are generated during normal fetal development. Proc Natl Acad Sci. 2002;99(12):8242-7. DOI:10.1073/pnas.112218799

46. Murray L, McCarron P, Bailie K, Middleton R, Davey Smith G, Dempsey S, et al. Association of early life factors and acute lymphoblastic leukaemia in childhood: historical cohort study. $\mathrm{Br} /$ Cancer. 2002;86(3):356-61. DOI: 10.1038/sj.bjc.6600012

47. Naumburg E, Bellocco R, Cnattingius S, Jonzon A, Ekbom A. Perinatal exposure to infection and risk of childhood leukemia. Med Pediatr Oncol. 2002;38(6):391-7. DOI:10.1002/mpo.10084

48. Neglia JP, Linet MS, Shu XO, Severson RK, Potter JD, Mertens AC, et al. Patterns of infection and day care utilization and risk of childhood acute lymphoblastic leukaemia. Br J Cancer. 2000;82(1);234-40. DOI:10.1054/bjoc.1999.0905

49. Nishi M, Miyake H. A case-control study of non-T cell acute lymphoblastic leukaemia of children in 
Hokkaido, Japan. I Epidemiol Community Health. 1989;43(4):352-5 .

50. Okcu MF, Goodman KJ, Carozza SE, Weiss NS, Burau KD, Bleyer WA, et al. Birth weight, ethnicity, and occurrence of cancer in children: a populationbased, incident case-control study in the state of Texas, USA. Cancer Causes Control. 2002;13(7):595-602. DOI:10.1023/A:1019555912243

51. Osterholm MT. Infectious disease in child day care: an overview. Pediatrics. 1994;94(6 Pt 2):987-90.

52. Perrillat F, Clavel J, Auclerc MF, Baruchel A, Leverger G, Nelken B, et al. Day-care, early common infections and childhood acute leukaemia: a multicentre French case-control study. Br J Cancer. 2002;86(7):1064-9. DOI:10.1038/sj.bjc.6600091

53. Perrillat F, Clavel J, Jaussent I, Baruchel A, Leverger G, Nelken B, et al. Breast-feeding, fetal loss and childhood acute leukaemia. Eur J Pediatr. 2002;161(4):235-7.

54. Petridou E, Kassimos D, Kalmanti M, Kosmidis H, Haidas S, Flytzani V, et al. Age of exposure to infections and risk of childhood leukaemia. BMJ. 1993;307(6907):774.

55. Petridou E, Trichopoulos D, Kalapothaki V, Pourtsidis A, Kogevinas M, Kalmanti M, et al. The risk profile of childhood leukaemia in Greece: a nationwide casecontrol study. Br J Cancer. 1997;76(9):1241-7.

56. Petridou E, Dalamaga M, Mentis A, Skalkidou A, Moustaki M, Karpathios T, et al. Evidence on the infectious etiology of childhood leukemia: the role of low herd immunity (Greece). Cancer Causes Control. 2001;12(7):645-52.

57. Petridou E, Trichopoulos D. Leukemias. In: Adami H-O, Hunter DJ, Trichopoulos D, editors. Textbook of cancer epidemiology. New York: Oxford University Press; 2002. p. 556-72.

58. Poole C, Greenland S, Luetters C, Kelsey JL, Mezei G. Socioeconomic status and childhood leukaemia: a review. Int J Epidemiol. 2006;35(2):370-84. DOI:10.1093/ije/dyi248

59. Randolph VL, Heath CW Jr. Influenza during pregnancy in relation to subsequent childhood leukemia and lymphoma. Am J Epidemiol. 1974;100(5):399-409.

60. Reis RS, Camargo B, Santos MO, Oliveira JM, Silva FA, Pombo-de-Oliveira MS. Childhood leukemia incidence in Brazil according to different geographical regions. Pediatr Blood Cancer. 2011;56(1):58-64. DOI:10.1002/pbc.22736

61. Reynolds P, Von Behren J, Elkin EP. Birth characteristics and leukemia in young children. Am J Epidemiol. 2002;155(7):603-13. DOI:10.1093/aje/155.7.603

62. Ribeiro KB, Buffler PA, Metayer C. Socioeconomic status and childhood acute lymphocytic leukemia incidence in São Paulo, Brazil. Int J Cancer. 2008;123(8):1907-12. DOI:10.1002/ijc.23738

63. Roman E, Watson A, Bull D, Baker K. Leukaemia risk and social contact in children aged 0-4 years in southern England. J Epidemiol Community Health. 1994;48(6):601-2.

64. Roman E, Simpson J, Ansell P, Kinsey S, Mitchell CD, Mckinney PA, et al. Childhood acute lymphoblastic leukemia and infections in the first year of life: a report from the United Kingdom Childhood Cancer Study. Am J Epidemiol. 2007;165(5):496-504. DOI:10.1093/aje/kwk039

65. Rosenbaum PF, Buck GM, Brecher ML. Early child-care and preschool experiences and the risk of childhood acute lymphoblastic leukemia. Am I Epidemiol. 2000;152(12):1136-44. DOI:10.1093/aje/152.12.1136

66. Rosenbaum PF, Buck GM, Brecher ML. Allergy and infectious disease histories and the risk of childhood acute lymphoblastic leukaemia. Paediatr Perinat Epidemiol. 2005;19(2):152-64. DOI:10.1111/j.1365-3016.2005.00634.x

67. Rudant J, Orsi L, Menegaux F, Petit A, Baruchel A, Bertrand Y, et al. Childhood acute leukemia, early common infections, and allergy: The ESCALE Study. Am J Epidemiol. 2010;172(9):1015-27. DOI:10.1093/aje/kwq233

68. Schlehofer B, Blettner M, Geletneky K, Haaf HG, Kaatsch P, Michaelis J, et al. Seroepidemiological analysis of the risk of virus infections for childhood leukaemia. Int / Cancer. 1996;65(5):584-90. DOI:10.1002/(SICI)10970215(19960301)65:5<584::AID-IJC5>3.0.CO;2-Zm

69. Schmiegelow K, Vestergaard T, Nielsen SM, Hjalgrim $\mathrm{H}$. Etiology of common childhood acute lymphoblastic leukemia: the adrenal hypothesis. Leukemia. 2008;22(12):2137-41. DOI:10.1038/leu.2008.212

70. Schüz J, Kaletsch U, Meinert R, Kaatsch P, Michaelis J. Association of childhood leukaemia with factors related to the immune system. Br J Cancer. 1999;80(34):585-90. DOI:10.1038/sj.bjc.6690395

71. Sehgal S, Mujtaba S, Gupta D, Aggarwal R, Marwaha RK. High incidence of Epstein Barr virus infection in childhood acute lymphocytic leukemia: a preliminary study. Indian J Pathol Microbiol. 2010;53(1):63-7. DOI:10.4103/0377-4929.59186

72. Shu XO, Linet MS, Steinbuch M, Wen WQ, Buckley JD, Neglia JP, et al. Breast-feeding and risk of childhood acute leukemia. J Natl Cancer Inst. 1999;91(20):1765-72. DOI:10.1093/jnci/91.20.1765

73. Shu XO, Han D, Severson RK, Chen Z, Neglia JP, Reaman $\mathrm{GH}$, et al. Birth characteristics, maternal reproductive history, hormone use during pregnancy, and risk of childhood acute lymphocytic leukemia by immunophenotype (United States). Cancer Causes Control. 2002;13(1):15-25. DOI:10.1023/A:1013986809917

74. Shurtleff SA, Buijs A, Behm FG, Rubnitz JE, Raimondi $\mathrm{SC}$, Hancock ML, et al. TEL/AML1 fusion resulting from a cryptic $\mathrm{t}(12 ; 21)$ is the most common genetic lesion in pediatric ALL and defines a subgroup of patients with an excellent prognosis. Leukemia. 1995;9(12):1985-9.

75. Simpson J, Smith A, Ansell P, Roman E. Childhood leukaemia and infectious exposure: a report from the United Kingdom Childhood Cancer Study (UKCCS). Eur J Cancer. 2007;43(16):2396-403. DOI:10.1016/j.ejca.2007.07.027

76. Smith M. Considerations on a possible viral etiology for B-precursor acute lymphoblastic leukemia of childhood. J Immunother.1997;20(2):89-100.

77. Tedeschi R, Bloigu A, Ogmundsdottir HM, Marus A, Dillner J, Depaoli P, et al. Activation of maternal Epstein-Barr virus 
infection and risk of acute leukemia in the offspring. Am J Epidemiol. 2007;165(2):134-7. DOI:10.1093/aje/kwj332

78. Till M, Rapson N, Smith PG. Family studies in acute leukaemia in childhood: a possible association with autoimmune disease. Br J Cancer. 1979;40(1):62-71.

79. UK Childhood Cancer Study Investigators. Breastfeeding and childhood cancer. $\mathrm{Br} /$ Cancer. 2001;85(11):1685-94. DOI:10.1054/bjoc.2001.2110

80. Urayama KY, Buffler PA, Gallagher ER, Ayoob JM, Ma $X$. A meta-analysis of the association between daycare attendance and childhood acute lymphoblastic leukaemia. Int J Epidemiol. 2010;39(3):718-32. DOI:10.1093/ije/dyp378

81. Urayama KY, Ma X, Selvin S, Metayer C, Chokkalingam $\mathrm{AP}$, Wiemels JL, et al. Early life exposure to infections and risk of childhood acute lymphoblastic leukemia. Int J Cancer. 2011;128(7):1632-43. DOI:10.1002/ijc.25752

82. Van Steensel-Moll HA, Valkenburg HA, Zanen GE. Childhood leukemia and infectious diseases in the first year of life: a register-based case-control study. Am J Epidemiol. 1986;124(4):590-4.

83. Vianna NJ, Polan AK. Childhood lymphatic leukemia: prenatal seasonality and possible association with congenital varicella. Am J Epidemiol. 1976;103(3):321-32.

84. Waly MI, Ali A, Al-Saadoon M, Al-Mukhaini YK, Wali YA. Breastfeeding is not associated with risk of developing childhood leukemia in the Sultanate of Oman. Asian Pac J Cancer Prev. 2011;12(8):2087-91.

85. Westergaard T, Andersen PK, Pedersen JB, Olsen JH, Frisch M, Sørensen HT, et al. Birth characteristics, sibling patterns, and acute leukemia risk in childhood: a population-based cohort study. I Natl Cancer Inst. 1997;89(13):939-47. DOI:10.1093/jnci/89.13.939

86. Wiemels J. Perspectives on the causes of childhood leukemia. Chem Biol Interact. 2012;196(3):59-67. DOI:10.1016/j.cbi.2012.01.007.

The authors declare that there are no conflicts of interest. 